\title{
El método micro-textual como herramienta de análisis de foros en línea en contextos multiculturales ${ }^{1}$
}

\section{The micro-textual method as a tool for analysis of online forums in multicultural contexts}

\author{
Marcelo I. Dorfsman \\ Universidad Hebrea de Jerusalem. Israel \\ marcelo.dorfsman@mail.huji.ac.il
}

\begin{abstract}
Resumen
En este trabajo presentaremos el método micro-textual, como una herramienta eficiente de análisis en la investigación que se está desarrollando sobre enseñanza en línea y multiculturalidad en la Educación Superior, en el Centro Melton de Educación Judía de la Universidad Hebrea de Jerusalem. El propósito del trabajo es mostrar de qué manera el método posibilita identificar y caracterizar diferentes tipos de diálogo en los foros en línea, y asimismo examinar su evolución.

El método está constituido por una batería conceptual desarrollada para el análisis de textos y su objetivo es contribuir a la comprensión del discurso textual e intertextual y su evolución en cursos virtuales, en particular en la enseñanza superior. El mismo utiliza componentes textuales y contextuales para la comprensión de textos, siendo un componente central el indicador etno-cultural, vinculado a la pertenencia del participante a un grupo religioso o cultural, y a problemáticas relacionadas con su identidad. En la ejemplificación elegida para este trabajo, propondremos analizar cuestiones vinculadas a la interrelación entre los indicadores personales, profesionales y los etno-culturales; así como también al rol del docente en la educación superior en línea.
\end{abstract}

Palabras clave: método micro-textual, enseñanza en línea, enseñanza multicultural.

\begin{abstract}
In this paper, we present the micro-textual method as an efficient analysis tool for the research that is being conducted about online teaching and multiculturalism in higher education, in Melton Centre for Jewish Education in the Hebrew University of Jerusalem.
\end{abstract}

\section{${ }^{1}$ Nota del autor}

Marcelo I. Dorfsman (Ph.D), Centro Melton de Educación Judía, Facultad de Educación, Universidad Hebrea de Jerusalem.

La presente investigación se realiza en el marco del programa de enseñanza mixta (Blended Learning) y cuenta con el apoyo del Centro Melton de Educación Judía.

El autor agradece la colaboración de los participantes del programa, quienes gentilmente han accedido a que sus textos sean utilizados con fines académicos, atendiendo a las normas éticas y de confidencialidad. El autor asimismo autoriza a utilizar los contenidos de este artículo, con la correspondiente cita bibliográfica.

Toda consulta sobre la misma dirigirla a Marcelo I. Dorfsman, Centro Melton de Educación Judía, Universidad Hebrea, Monte Scopus (91905). 
The aim of this paper is to show how the method allows to identify and characterize different types of dialogues in online forums, and to examine their evolution.

The method consists of a toolbox of terms that was developed for the analysis of texts. The aim of the method is to contribute to the understanding of textual and intertextual discourse and its evolution in virtual courses, particularly in higher education. This method uses textual and contextual components for the understanding of texts. The ethno-cultural indicator is a main component and it is related to the participant's membership in a religious or cultural group, and to issues related to its identity. Through the example that was chosen for this work, we propose to analyze questions related to the interrelationship among personal, professional and ethno-cultural indicators, as well as the role of the teacher in online higher education.

Keywords: micro-textual method, online teaching, multicultural education.

\section{Introducción}

En este trabajo presentaremos el método micro-textual, que es utilizado actualmente en una investigación que se está desarrollando en el Centro Melton de Educación Judía de la Universidad Hebrea de Jerusalem, en el campo de la enseñanza en línea y la multiculturalidad en la Educación Superior. Nuestro propósito es presentar este método de investigación como herramienta de análisis de las interacciones interpersonales e interculturales en el entorno virtual, ejemplificar en forma preliminar el modo en que es posible identificar y caracterizar diferentes tipos de diálogo y su evolución en los foros en línea de un curso virtual, e identificar diferentes tipos de interacción en dicho entorno virtual.

El método ha sido desarrollado en la disertación doctoral del autor del artículo (Dorfsman, 2012a), actualizado en el marco de diferentes investigaciones desarrolladas en el campo de la educación museal y transnacional (Dorfsman, 2012b; Dorfsman \& Horenczyk, 2014; Dorfsman \& Horenczyk, 2016), e investigaciones que analizan la enseñanza y la enseñanza en línea desde una perspectiva cultural (Gunawardena, Wilson, \& Nolla, 2003; Jung, Gunawardena, \& Moore, 2014; Schachter \& Galili-Schachter, 2012). El objetivo del método es contribuir a la comprension del discurso textual e intertextual, y su evolución en los cursos virtuales de la Enseñanza Superior ${ }^{1}$.

Este trabajo es parte de una investigación que se desarrolla actualmente en un programa de M.A. (Maestría) en Educación Judía en modalidad Blended Learning, que se inició en el mes de Marzo de 2016, con veinte estudiantes de nueve países del mundo. En el mismo los estudiantes participan de siete cursos en línea en dos semestres y un seminario presencial de verano. Los cursos en línea se dictan en la modalidad asincrónica de acuerdo con un modelo pedagógico que privilegia los foros de discusión.

El método micro-textual como herramienta de análisis de foros en línea en contextos multiculturales. Marcelo I. Dorfsman 


\section{Marco Teórico}

La revolución tecnológico-digital impactó notoriamente en el campo de la enseñanza y el aprendizaje (Avidov-Ungar \& Eshet-Alkalai, 2014; Kale \& Goh, 2014; Youssef, Dahmani, \& Omrani, 2015). En el marco de la misma, surgieron investigaciones en campos diversos como: los procesos de enseñanza y aprendizaje en los entornos virtuales; investigaciones comparativas entre la enseñanza en línea y la enseñanza presencial; la interacción en los foros en línea, y las concepciones docentes acerca de la enseñanza virtual (Hung, 2012; Youssef et al., 2015).

En los últimos veinte años creció el campo de la investigación en torno del significado del entorno virtual y su influencia sobre los procesos de la alfabetización y de la alfabetización digital. Leu (2004) destacó el rol de la alfabetización crítica, sosteniendo que existe una relación mutua entre los procesos de alfabetización y los entornos tecnológicos. Un desarrollo adicional en el campo se produjo con las investigaciones que se ocupan de los aspectos culturales en los entornos virtuales, en el marco de las cuales se analiza la influencia de los elementos culturales (nacionalidad, género, tradiciones, valores, etc.) sobre las concepciones de aprendizaje y los procesos de enseñanza en los entornos virtuales (Gunawardena et al., 2003; Jung et al., 2014; Shattuck, 2005). En el contexto de esta nueva modalidad de enseñanza, Schachter \& Galili-Schachter (2012) indagan en su trabajo acerca de diferentes tipos de alfabetización - en particular la crítica y la cultural. Su trabajo se centra en el análisis de la enseñanza de materias humanísticas basadas en textos y en particular en textos canónicos. El interés que despierta el mismo se cifra en la tercera dimensión que proponen los autores, como adición a las ya existentes; la dimensión identitaria. La dimensión identitaria está vinculada con los procesos de enseñanza en los cuales el docente se propone explícitamente utilizar los textos con el fin de desarrollar y consolidar la identidad de sus alumnos; a esta práctica del docente la denominan alfabetización identitaria. Los autores abren de esta manera un campo interesante para investigaciones interesadas en analizar aspectos que hacen a la educación identitaria en un entorno multicultural.

En esta línea, Dorfsman y Horenczyck (2016) han propuesto - en el campo de la educación museal - una actualización del marco conceptual de Dierking \& Falk (1992) compuesto por tres contextos; el personal, el físico y el social. En la actualización se propone el contexto tecnológico y el etno-cultural, que incluye los aspectos étnicos e identitarios del proceso educativo. De este modo, el análisis del contexto educativo se enriquece con una nueva perspectiva, que es el mutuo impacto entre los componentes culturales e identitarios del sujeto que aprende y su interrelación con los componentes culturales e identitarios del contexto en el cual aprende. El investigador se preguntará, en este contexto ¿En qué medida la práctica docente considera rasgos étnicos y culturales de sus alumnos? ¿En qué medida el contexto favorece u obstaculiza un espacio de encuentro multicultural? ¿Cuál es el lugar que ocupa - si es que lo hace - el desarrollo de la identidad

El método micro-textual como herramienta de análisis de foros en línea en contextos multiculturales. Marcelo I. Dorfsman 
en la práctica docente? Estos interrogantes no siempre están presentes en el discurso pedagógico, siendo que los avances identificados en los diferentes campos mencionados pueden constituir un interesante aporte para su desarrollo.

\section{El método micro-textual}

El método micro-textual pertenece a la familia de los métodos de la investigación cualitativa; está basado en el análisis de textos ("posts") que producen los participantes de los foros en línea y su objetivo es analizar el tipo de discurso que se produce y su evolución a lo largo de un curso virtual, mediante criterios de identificación, clasificación y codificación (Dorfsman, 2012a). Este método se basa en la Teoría de la Acción ${ }^{2}$ (Fine, 1995; Pickering \& Ferreira, 2008); el modelo metodológico corresponde al enfoque basado en procesos (Wagoner, 2008), y el nivel de análisis es el microgenético.

El nivel microgenético analiza conductas y procesos cognitivos que se producen durante lapsos cortos - microsegundos, segundos, minutos, horas o semanas - con el fin de comprenderlos y justificarlos. Este nivel, que se diferencia del filogenético y el ontogenético, es utilizado para analizar mediciones en ciencias como la Física, la Química y la Biología. En nuestra perspectiva, el nivel microgenético nos permite visualizar procesos y modificaciones que se dan en plazos breves y que que son reflejados en los textos que se producen en la práctica de la enseñanza en línea.

El objeto de esta investigación en proceso es el discurso que se desarrolla en los foros interactivos de un curso virtual, considerando cuatro contextos: el personal, el social, el profesional y el etno-cultural (Dierking \& Falk, 1992; Dorfsman \& Horenczyk, 2016; Falk \& Dierking, 2008). El material de la investigación son los textos de los foros, que reflejan el proceso de enseñanza que se desarrolla en las clases y su evolución a lo largo del período. El análisis se lleva a cabo en las cuatro etapas del método micro-textual: definiciones generales; modelización, secuencia de significados y líneas teóricas.

\section{Definiciones Generales}

En esta primera etapa, se analizan las características generales del fenómeno a investigar - en este caso los cursos virtuales y el programa en el que están insertos - se definen sus particularidades, el contexto en el que se desarrollan, el proceso de diseño instruccional que les dio origen, sus componentes y características.

Como decimos al comienzo, nuestra intención es analizar la práctica de enseñanza de los docentes de siete cursos en línea de un programa de Maestría en modalidad Blended Learning. En el programa participan veinte alumnos, de nueve países del mundo y el objeto de análisis son los foros de discusión en línea del programa.

\section{Modelización}

En esta etapa se modeliza el proceso de indagación del método micro-textual, y el proceso de interpretación que se lleva a cabo en el proceso de investigación. Esta etapa se sustenta en un doble proceso de análisis - longitudinal y transversal - a lo largo del cual se

El método micro-textual como herramienta de análisis de foros en línea en contextos multiculturales. Marcelo I. Dorfsman 
hará uso de una primer batería de herramientas conceptuales; los indicadores de contenido textuales y contextuales (Dorfsman, 2012). En el análisis longitudinal, se lleva a cabo el análisis de los foros en línea, a lo largo de una clase y luego, a lo largo de la secuencia clases de un mismo curso. Este tipo de análisis requiere de una lectura intensa y profunda de los foros de discusión de un curso, de acuerdo con una secuencia cronológica. Este análisis se caracteriza por llevarse a cabo mientras se desarrollan núcleos temáticos de una misma disciplina; entonces, el foco puede centrarse en el interjuego de conceptos y terminologías; en actitudes de los participantes respecto de los mismos contenidos, en el análisis de la coherencia de la práctica docente y su impacto en la clase, etc.

El análisis transversal es un análisis comparativo de discusiones paralelas que se desarrollan en dos o tres cursos en línea que se imparten en forma simultánea al mismo grupo de alumnos En este caso, las lineas discursivas serán diferentes que en el anterior dado que las temáticas disciplinares lo son; pero entonces se pueden identificar recurrencias en modalidades de participación de los alumnos, se pueden establecer diferentes tipos de respuestas de docentes en situaciones similares, se pueden comparar estilos de participación en diferentes circunstancias, y ante diferentes desafíos, etc.

\section{Herramientas conceptuales: indicadores de contenido}

El doble proceso de análisis que se lleva a cabo requiere de una batería de herramientas conceptuales para el análisis de textos. Estas herramientas, que desarrollaremos en adelante, son: los indicadores textuales - que refieren al análisis de los textos propiamente dichos; los indicadores contextuales - que dan cuenta de las mutuas interrelaciones entre el sujeto y el texto; los "eventos de habla" - expresiones particulares que dan cuenta de situaciones particulares del lenguaje; y las "palabras llave", palabras que - por su modalidad de aparición y reiteración en el texto - asumen un significado particular.

Desde nuestra perspectiva, los textos son la "materia prima" en la cual el investigador intenta identificar las huellas del desarrollo del diálogo y de la interacción en línea. Nos referiremos entonces a los indicadores de contenido, como aquellos que se pueden identificar en los textos en formatos diferentes; observaciones, preguntas, reacciones, comentarios, etc. Tal lo adelantado, en el método micro-textual se han definido dos tipos de indicadores:

El método micro-textual como herramienta de análisis de foros en línea en contextos multiculturales. Marcelo I. Dorfsman 


\section{Indicadores textuales}

Son aquelos que se refieren al texto en sí, y que pueden categorizarse como indicadores lngüísticos, disciplinares y tecnológico-multimediales (Schwab, 1961; Gudmundsdottir \& Shulman, 1987; Mishra \& Koehler, 2006; Shulman, 1999).

Un indicador lingüístico da cuenta de correcciones de lenguaje o referencias a las características del idioma. En nuestro programa, por ejemplo, participan estudiantes para los cuales el inglés no es lengua materna, por lo cual en muchos casos aparecen "disculpas" por posibles errores en su expresión escrita.

Un indicador disciplinar es el que hace mención a cuestiones propias de la disciplina en cuestión; cuando por ejemplo se aclara el significado de un concepto, o la modalidad de interpretación de un texto bíblico de acuerdo a una corriente determinada.

Un indicador tecnológico-multimedial es el que hace alusión a aspectos técnicos del curso, dificultades con la plataforma u otras herramientas disponibles (Google Drive, Web 2.0) o bien alusiones a recursos multimediales utilizados por docentes o alumnos.

\section{Indicadores contextuales}

Son aquellos que dan cuenta de las mutuas interrelaciones entre el texto y el sujeto que lo produce (Dorfsman, 2012). Estos indicadores pueden categorizarse como personales, sociales, profesionales y etno-culturales. Los personales refieren a la historia y vivencias personales del sujeto (Shkedi \& Horenczyk, 1995; Shkedi \& Nisan, 2006); los sociales refieren a la interacción que se da en el grupo; los profesionales refieren, en este caso $^{3}$, al rol profesional del educador y al modo en que el curso impacta en su visión y práctica de la educación y viceversa (Schön, 1987; Domingo, 1997); por último, los indicadores etnoculturales refieren a la pertenencia del sujeto a una comunidad cultural o religiosa, y dan cuenta entre otras, de cuestiones vinculadas a procesos de conformación de la identidad, analogías entre el entorno cultural propio y el del grupo, impacto del propio entorno cultural en la comprensión de los materiales estudiados e influencia de éstos en la percepción de sus propios entornos culturales (Dierking \& Falk, 1992; Dorfsman \& Horenczyk, 2016; Falk \& Dierking, 2008; Schachter \& Galili-Schachter, 2012).

En el programa que investigamos, el análisis de los indicadores etno-culturales es uno de nuestros objetos centrales dado que, como hemos dicho al principio, el grupo está compuesto por estudiantes provenientes de diferentes grupos étnicos, religiosos y nacionales, lo cual se pone de manifiesto en sus intervenciones; por ejemplo, al comparar textos y exégesis judíos con textos y exégesis cristianos; o bien cuando se comparan sistemas educativos en Israel y en China o India. Las evocaciones de los estudiantes aportan valor e interés a los foros de discusión.

El método micro-textual como herramienta de análisis de foros en línea en contextos multiculturales. Marcelo I. Dorfsman 


\section{Secuencia de Significados}

El proceso de análisis va generando un entramado intertextual que da cuenta de nuevos significados. Esta secuencia se expresa en diferentes tipos de articulación entre los textos, articulación en la cual los indicadores de contenido se resignifican y cobran nuevos sentidos. En el proceso de construcción de la secuencia de contenidos, se utilizan dos herramientas conceptuales: los "Eventos del habla" y las "Palabras-llave".

\section{Eventos del Habla}

Un "evento del habla", es un fenómeno que transcurre en el espacio textual y produce un cambio en el desarrollo del discurso (Catriel, 1999). En un evento del habla, hay diálogo y sentido, hay acuerdos y desacuerdos. Puede ocurrir en los diferentes niveles del discurso, y da cuenta de una modalidad específica de interacción entre los protagonistas de los diálogos en línea.

Por ejemplo, en uno de los foros, un estudiante menciona en su discurso la expresión "bar mitzvah factory", con la cual se refiere al fenómeno por el cual esta ceremonia religiosa "se industrializó" - considerando todas las implicancias sociales y económicas del caso. Esta expresión, que "resonó" en la mente de otra estudiante, impactó en la dirección del foro y en su contenido. Por este motivo es que la denominamos "evento de habla".

\section{Palabras "llave"}

Se trata de palabras que se repiten en sus diferentes variaciones ${ }^{4}$ a lo largo de una clase y de un curso, y sirven de "puente" de significados entre los diferentes eventos que van ocurriendo (Catriel, 1999; Ortner, 1973; Searle, 1989). Las palabras-llave se instalan como un "testimonio" de la evolución del diálogo en línea, y del proceso dinámico que acompaña su desarrollo. La palabra-llave es una herramienta central para la comprension de la "secuencia de significados", esto es, para la construcción de nuevas síntesis conceptuales producto del proceso de doble análisis transversal y logitudinal.

La palabra "value" por ejemplo, en una de las líneas de discusión, se convierte en una palabra-llave que utilizan con diferentes formatos, los estudiantes y el docente.

\section{Líneas teóricas}

El proceso de análisis que se desarrolla en la etapa anterior constituye una primera síntesis conceptual, a través de la cual se podrán identificar primeras tendencias en el desarrollo del discurso en línea de cada uno de los cursos analizados.

En la etapa de las líneas teóricas, volvemos al análisis de todos los cursos de un programa, en forma integral y comparativa. Tomando en cuenta los principios de la concepción holística, el modelo nos lleva a revisar nuevamente el programa en su totalidad

El método micro-textual como herramienta de análisis de foros en línea en contextos multiculturales. Marcelo I. Dorfsman 
para formular lineas teóricas generales que puedan explicar modelos de desarrollo de los diálogos en los foros de discusión en línea.

Al finalizar el proceso, las lineas teóricas contribuirán a obtener un panorama más claro del proceso de construcción del discurso en los foros virtuales, y nos ofrecerá nuevas perspectivas para la continuidad de la investigación.

\section{Un ejemplo de análisis micro-textual}

En este último parágrafo, ejemplificaremos el uso del método micro-textual. Lo haremos utilizando el foro de la clase 4 de uno de los cursos ddel programa, clase asincrónica que se llevó a cabo en la cuarta semana del mismo. En el ejemplo, mostraremos básicamente el análisis longitudinal del foro, haciendo mención a algunos de los conceptos centrales del modelo - indicadores de contenido, interacción abierta y oculta, evento de habla y palabra-llave ${ }^{5}$.

\section{Primer interjuego entre componentes, y un "evento de habla"}

A continuación presentamos una secuencia del foro 4 del curso seleccionado. Para presentar la secuencia, hemos seleccionado algunos textos que consideramos interesantes, y hemos alternado nuestros comentarios y análisis. El lector se encontrará con los extractos del foro recuadrados, y nuestros comentarios alternados.

En esta secuencia participan ${ }^{6}:$ El docente, con mucha experiencia en la enseñanza pero novato en la enseñanza en línea. M., estudiante joven, educadora en la educación no formal; J., estudiante veterana, educadora no formal; Sh, rabino ortodoxo; N., pastor evangelista; X., joven estudiante china; B, educadora formal, edad mediana.

En la apertura del diálogo, al presentar la problemática de la visión educativa, el docente procura integrar dos componentes; el personal y el profesional.

Students will explore the problems that they have in their professional lives in negotiating their educational visions with the visions of the educational institutions in which they practice. They will also discuss the writing assignment.

\section{Post \#1 ${ }^{7}$ :}

M. abre el diálogo...

I feel that this class has been incredibly beneficial to me, because I work in several different shuls as a "Hebrew School teacher" and none of the synagogues have a vision. Not a single one, out of five educational institutions! Meanwhile my educational vision is something similar to RAVSAK's (...) I've said it several times, I work at "1 bar mitzvah factory" synagogues (M).

El método micro-textual como herramienta de análisis de foros en línea en contextos multiculturales. Marcelo I. Dorfsman 


\section{Post \#2:}

M.., your phrase, "Bar Mitzvah factories" really resonated with me. Over and over I hear friends say that the ceremony was just that: a ritual to get through and, once done, it no longer had any meaning... (J)

... y J. lo continúa casi de inmediato:

M. es una educadora joven, y J. es veterana; ambas trabajan en educación judía no formal. La cuestión personal-profesional se transforma rápidamente en un texto crítico con ayuda del indicador etno-cultural e identitario; la expresión "Bar Mitzvah factories" no solamente lleva el diálogo a una crítica a la ceremonia y a su esencia tal como se realiza, sino que M. la utiliza como una manera de clasificar sinagogas - cada una y su ceremonia ${ }^{8}$. El uso de la palabra "factories" en plural ${ }^{9}$, insinúa que existen una serie de sinagogas que actúan de esta manera. Estos dos post son un ejemplo de interacción abierta entre las protagonistas.

Dada su incidencia en el desarrollo posterior de la secuencia, consideraremos a la expresión "Bar-Mitzvah factories" como un "evento de habla".

\section{Post \#3:}

My teaching is within Church institutions in the United States. Churches are complex houses of hierarchy and can be challenging to negotiate. I have simultaneously taught the same material at very liberal denominations as well as very fundamental Churches $(N)$

La respuesta de $\mathrm{N}$.

$\mathrm{N}$ abre su post relatando acerca de su práctica en iglesias (y no en sinagogas ${ }^{10}$ ). Al igual que sus compañeras, N. menciona las diferentes denominaciones de las iglesias en su país; si bien no se refiere directamente a ellas, es evidente que existe acá una interacción que parece ser una "interacción oculta".

$\mathrm{N}$. en su respuesta, procura referir al post del docente y remitirse al aspecto personal-profesional, pero rápidamente deriva en cuestiones etno-culturales:

.. How can you teach to both groups each week? Did I change my approach to the lesson to fit the ideology of the groups? No. God's truth is applicable to both (N).

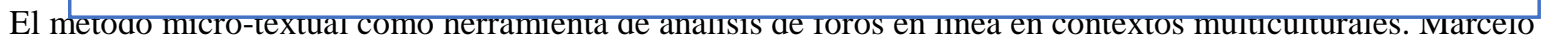




\section{Post \#4:}

En su primera respuesta al foro, el docente procura recuperar en el diálogo la preeminencia del indicador profesional:

... N is firm in his conviction that his educational-theological vision should be implemented vis-a-vis each Christian group that he teaches in an uncompromising and undiluted manner (although in the context of this class there was some hesitation and perhaps this is an example of sensitivity to the context)...

A partir de este primer análisis, surge la pregunta acerca de la contradicción aparente entre el indicador profesional y el etno-cultural en los foros de discusión, y nos preguntamos además si existe una tendencia entre determinados docentes a dar preeminencia a uno u a otro.

\section{La palabra-llave}

Continuando con la secuencia seleccionada en el foro 4, presentaremos ahora un ejemplo de cómo aparece y se desarrolla la palabra llave.

\section{Post \#5:}

Sh, se dirige directamente a N:

Like $N$, I also struggled with how to share my message with the students without compromising on my educational beliefs and personal values in the process. I found that the simplest way to approach this topic was not to change or alter the message/text itself but rather the delivery... (SH)

En su mensaje, Sh. expresa las preocupaciones compartidas entre ambos estudiantes, que es el vínculo entre sus creencias educacionales y sus valores personales. Los valores, se entiende que para ambos, son culturales y religiosos (religiones diferentes cuyo puente se expresa en la palabra "valores"). Por esto y por lo que vendrá después, definiremos a la palabra "value" como palabra-clave.

\section{Post \#6:}

J., la educadora veterana, abre ahora un post relatando una historia acerca de una sinagoga-escuela.

El método micro-textual como herramienta de análisis de foros en línea en contextos multiculturales. Marcelo 
I visited the synagogue's day school and was delighted with what I saw. The children were happy, and so were the teachers and the aides. The atmosphere was vibrant. However, when my son decided to enroll his children, he was told he'd be put on a wait list. Disappointed, we started to go home when two families, one Protestant, the other Catholic, came to pick up their children. We started talking and I asked them why they decided to enroll their students here. Both families gave the same answer: values. This was their priority $(\mathrm{J})$.

Como en la secuencia del foro, en la que el Sh. y N. coinciden en la palabra "values", también en el relato, dos familias; una católica y la otra protestante, coinciden con la judía en los "valores" que transmite la escuela (que eligieron o quieren elegir). También el relato de J. se inició en una referencia profesional, pero pasó rápidamente a la cuestión etno-cultural. La palabra "values" se afirma como palabra-llave en este foro.

\section{¿Cómo se detecta el aprendizaje mediante el método micro-textual?}

\section{Post \#7:}

"X, aprende en la red".

A diferencia de la mayoría de sus compañeros, $\mathrm{X}$ carece de experiencia profesional:

I actually do not have much to share on the topic of this week as I have no experience working in an institution as an educator, but I did read what other students have posted here carefully trying to draw something from their experience, which is really inspiring due to the diversity of the professions and background of every students in this course $(X)$.

Por ese motivo, ella propone "extraer parte de la experiencia de sus compañeros", con las cuales propondrá un interesante resumen conceptual de lo dicho hasta ese momento en el foro. En su post, la palabra "diversity" equivale en cierto modo a la palabra "values" por el tinte multicultural que asume el foro. Pero lo más interesante es comparar este post con un post de esta misma alumna que aparece la semana anterior en otro curso:

\section{Post \#8:}

Since I have no teaching experience yet, I cannot say which is more salient in my teaching. However according to my past experience of reading the Biblical stories...(X).

También acá $\mathrm{X}$ abre el post indicando que no tiene experiencia docente, pero a diferencia del post \#7, intentará cubrir su carencia con su experiencia como alumna. Es en este punto que surge el siguiente interrogante: ¿De qué manera el análisis micro-textual da cuenta de las estrategias de aprendizaje de los alumnos y de su evolución a lo largo de las clases?

Efectivamente, parecería que entre el Post \#7 y el \#8, hay un cambio de estrategia ante situaciones similares, siendo que la segunda (\#7) parece ser más sofisticada y elaborada que la primera; mientras que en $\# 8, \mathrm{X}$ acude a su experiencia merced a un

El método micro-textual como herramienta de análisis de foros en línea en contextos multiculturales. Marcelo I. Dorfsman 
proceso autorreflexivo y metacognitivo, en \#7, debe reconstruir experiencias de otros, articularlas entre sí y darles forma de relato unificado para lo cual deberá sostener su propia experiencia como anclaje.

\section{El componente personal en los foros de discusión}

\section{Post \#9:}

Hacia el final del foro, B. publica el siguiente post en el cual exterioriza su actual conflicto $^{11}$ :

The conflicts I have in my professional lives are actually based on my personal life (...) Where is my place here as an educator and as a parent to a kid goes to this school? My dreams changed (...) it's still complicated for me to make a connection between "the principal of (xxx) citizenship" and Jewish values. I have no doubt that the school I work for does a lot to strengthen the connection with Israel as I'm part of the people that responsible for it, but this is a big philosophical question that means for me to look at my goals as I'm trying to develop my vision and to check is my vision have ideals that design my Jewish Identity and that are very dynamic and does it emphasize what the school ask to accomplish? (BA)

Si bien el indicador que utiliza es claramente el personal, B lo articula con su vida profesional y exterioriza un conflicto que afecta a su identidad judía e israelí. La palabrallave, "values", regresa al centro discursivo del foro.

\section{El docente y los indicadores de contenido de los foros virtuales:}

\section{Post \#10:}

El docente del curso resume la discusión como una confrontación entre dos valores, los estudios seculares frente a los estudios judaicos.

These two values at times clash. For example, schools struggle with the question: How much time do we devote to secular studies and how much time do we devote to Jewish studies? It also relates to the message that we impart to our students (both implicitly and explicitly) in terms of what is really important.

El resumen del docente, tal cual parece ser su estilo, es netamente profesional. Él vuelve a la cuestión de la enseñanza en las escuelas, muestra dos situaciones y explica cuáles pueden ser las proyecciones de un mensaje o de otro. La palabra-llave valores cierra esta discusión. La misma ha recorrido un largo camino desde su aparición, sirviendo por momentos para mostrar ideas coincidentes (en los post \#6 y \#7), pero también opuestas y confrontantes (post \#9 y \#10).

El método micro-textual como herramienta de análisis de foros en línea en contextos multiculturales. Marcelo 
El docente se muestra coherente a lo largo de sus tres intervenciones, sosteniendo la impronta netamente profesional que quiso imprimirle al foro desde un principio, y finaliza en esa misma postura. Cabe preguntarse en qué medida la elección de una perspectiva (personal, profesional o etno-cultural) puede caracterizar el perfil y la práctica de los educadores - sea en entornos digitales o presenciales $-\mathrm{o}$ bien de los estudiantes.

El método micro-textual como herramienta de análisis de foros en línea en contextos multiculturales. Marcelo 


\section{A modo de síntesis}

En este trabajo, hemos presentado el método micro-textual, como un método de análisis de textos a utilizarse en la investigación de la enseñanza en línea en contextos multiculturales. El mismo se caracteriza por la utilización de indicadores textuales y contextuales para la comprensión de textos y su desarrollo, incluyendo el indicador etnocultural que se vincula con la pertenencia del participante a un grupo étnico, cultural o religioso, y por ende a cuestiones de identidad.

En la ejemplificación, se pueden apreciar las discusiones signadas en forma alternada por indicadores personales, profesionales y etno-culturales; hemos indicado la existencia de un "evento de habla" (Bar Mitzva Factory-factories), que da cuenta de la transición del discurso profesional al ento-cultural y religioso; y de una palabra-llave (values) que ha sido utilizada en forma alternada para dar cuenta de unidad y de confrontación de valores.

Hemos señalado también la aparición del indicador profesional en la estudiante X, en la cual se exteriorizan estrategias de aprendizaje y una posible evolución de las mismas; y del indicador personal en la estudiante $B$, que exterioriza el conflicto existencial al que está expuesta.

Para finalizar, queremos destacar los dos tipos de interacción - abierta y oculta que se aprecian en los foros; nos preguntamos acerca de la potencia e influencia de cada una de ellas. La existencia de una palabra-llave que se reitera a lo largo del foro parece reforzar la interacción oculta entre los participantes.

Algunas de las preguntas que surgen de este análisis preliminar: ¿Cuál es el rol y el impacto del indicador etno-cultural en la dinámica de los foros y en las prácticas de enseñanza de los docentes? ¿Es posible determinar perfiles docentes y de estudiantes a partir de estos indicadores? ¿Hasta qué punto las herramientas del método son suficientes para analizar los textos y el discurso personal, profesional y etno-cultural que se desarrolla en los entornos digitales?

Confiamos en que la profundización del trabajo de investigación que se inicia aportará a la comprensión de la práctica educativa en los foros en línea y contribuirá al enriquecimiento de la didáctica en general.

Fin de redacción del artículo: 11 de noviembre de 2016

Fecha de aprobación: 19 de octubre de 2016 Fecha de edición: 15 de noviembre de 2016

El método micro-textual como herramienta de análisis de foros en línea en contextos multiculturales. Marcelo I. Dorfsman 
Dorfsman, M. (2016). El método micro-textual como herramienta de análisis de foros en línea en contextos multiculturales. RED. Revista de Educación a Distancia, 51. Consultado el (dd/mm/aaaa) en http://www.um.es/ead/red/51/

\section{Bibliografía}

Avidov-Ungar, O., \& Eshet-Alkalai, Y. (2014). TPACK Revisited: A Systemic Perspective on Measures for Predicting Effective Integration of Innovative Technologies in School Systems. Journal of Cognitive Education and Psychology, 13(1), 19-31 .

Catriel, T (1999) Palabras Llave: patrones culturales y comunicación en Israel. Universidad de Haifa $^{12}$.

Collier, S ,.Foley, B., Moguel, D., \& Barnard, I. (2013). Write for Your Life: Developing Digital Literacies and Writing Pedagogy in Teacher Education. Contemporary Issues in Technology and Teacher Education, 13(3), 262-284 .

Dierking, L. D., \& Falk, J. H. (1992). Redefining the Museum Experience: The Interactive Experience Model .

Diriwächter, R., \& Valsiner, J. (Eds.) (2008). Striving towards the whole. New Brunswick, N.J: Transaction Publishers.

Domingo, J. C. (1997). La autonomía del profesorado: Ediciones Morata, Madrid.

Dorfsman, M. (2012a). The evolution of teachers' pedagogical thinking in the process of preparing on-line courses for Jewish education in the Diaspora. (Doctoral Disertation), Hebrew University of Jerusalem, Jerusalem.

Dorfsman, M. (2012b). La profesión docente en contextos de cambio: el docente global en la sociedad de la información. [http://www.um.es/ead/reddusc/6]. RED-DUSC. Revista de Educación a Distancia-Docencia Universitaria en la Sociedad del Conocimiento, 6 .

Dorfsman, M., \& Horenczyk, G. (2014). La dimensión ideológico-identitaria en un proyecto multicultural de educación museal. RED, Revista de Educación a Distancia: Número monográfico sobre" Interculturalidad en el nuevo paradigma educativo, 15 .(41)

Dorfsman, M. y Horenczyk, G. (2016). Desarrollo de un museo educativo interactivo para la comunidad judía de Chile: aspectos epistemológicos, pedagógicos y tecnológicos, (en hebreo, en prensa).

Falk, J. H., \& Dierking, L. D. (2008). Enhancing visitor interaction and learning with mobile technologies. In L. T. a. K. Walker (Ed.), Digital technologies and the museum experience: Handheld guides and other media (pp. 19-33). Plymouth UK: AltaMira Press.

Fine, G. (1995). Public narration and group culture: discerning discourse in social movements. Social movements and culture, 127-143 .

El método micro-textual como herramienta de análisis de foros en línea en contextos multiculturales. Marcelo I. Dorfsman 
Garrod, S., \& Pickering, M. (2004). Why is conversation so easy? Trends in Cognitive Sciences, 8(1), 8-11.

Gudmundsdottir, S., \& Shulman, L. (1987). Pedagogical content knowledge in social studies. Scandinavian Journal of Educational Research, 31(2), 59-70 .

Gunawardena, C. N., Wilson, P. L., \& Nolla, A. C. (2003). Culture and online education. Handbook of distance education, 753-775 .

Hung, J. 1. (2012). Trends of e-learning research from 2000 to 2008: Use of text mining and bibliometrics. British Journal of Educational Technology, 43(1), 5-16 .

Jung, I., Gunawardena, C. N., \& Moore, M. G. (2014). Culture and online learning: Global Perspectives and Research .

Kahiigi Kigozi, E ,.Ekenberg, L., Hansson, H., Tusubira, F., \& Danielson, M. (2008). Exploring the e-learning state of art. Electronic Journal of e-learning, 6(2), 77-88 .

Kale, U., \& Goh, D. (2014). Teaching style, ICT experience and teachers' attitudes toward teaching with Web 2.0. Education and Information Technologies, 19(1), 41-60 .

Kleine-Horst, L. (2008). From visual actual genesis and ontogenesis toward a theory of man. In E. Abbey \& R. Diriwächter (Eds.), Innovating genesis: microgenesis and the constructive mind in action (pp. 3-40). Charlotte, North Carolina: Information Age Publishing, Inc.

Leu, D. J., Kinzer, C. K., Coiro, J. L., \& Cammack, D. W. (2004). Toward a theory of new literacies emerging from the Internet and other information and communication technologies. Theoretical models and processes of reading, 5, 1570-1613 .

Mishra, P., \& Koehler, M. (2006). Technological pedagogical content knowledge: A framework for teacher knowledge. Teachers College Record, 108(6), 1017-1054 .

Ortner, S. B. (1973). On Key Symbols. American Anthropologist, New Series, 75(5) 13381346.

Pickering, M., \& Ferreira, V. (2008). Structural priming: A critical review. Psychological bulletin, 134(3), 427.

Schachter, E. P., \& Galili-Schachter, I. (2012). Identity literacy: Reading and teaching texts as resources for identity formation. Teachers College Record, 114 .(5)

Searle, J. R. (1974). Speech acts: An essay in the philosophy of language. Cambridge, Melbourne, Madrid: Cambridge University Press.

Shattuck, K. (2005 .(Cultures meeting cultures in online distance education: Perceptions of international adult learners of the impact of culture when taking online distance education courses designed and delivered by an American university. Unpublished doctoral dissertation). The Pennsylvania State University .

Shkedi, A. (2001). Studying culturally valued texts: teachers' conception vs. students' conceptions. Teaching and Teacher Education, 17(3), 333-347.

Shkedi, A., \& Horenczyk, G. (1995). The role of teacher ideology in the teaching of culturally valued texts. Teaching and Teacher Education, 11(2), 107-117.

El método micro-textual como herramienta de análisis de foros en línea en contextos multiculturales. Marcelo I. Dorfsman 
Shkedi, A., \& Nisan, M. (2006). Teachers' Cultural Ideology: Patterns of Curriculum and Teaching Culturally Valued Texts. Teachers College Record, 108(4), 687-725.

Shulman, L. (1986). Those who understand: Knowledge growth in teaching. Educational researcher, 15(2), 4 .

Shulman, L. (1999). Knowledge and teaching: Foundations of the new reform. Learners and pedagogy, 61-77.

Wagoner, B. (2008). Developing "Development" in Theory and Method. A commentary on Kleine-Horst (2007). In E. Abbey \& R. Diriwächter (Eds.), Innovating genesis: microgenesis and the constructive mind in action (pp. 41-63). Charlotte, North Carolina: Information Age Pub Inc.

Wood, A. F., \& Smith, M. J. (2004). Online communication: Linking technology, identity, \& culture: Routledge.

Youssef, A. B., Dahmani, M., \& Omrani, N. (2015). Information technologies, students'eskills and diversity of learning process. Education and Information Technologies, 20(1), 141-159 .

$1 \quad$ El método se describe en la tercera sección del artículo, pág. 8.

2 Action Theory

3 El curso que se investiga es una Maestría en Educación Judía.

4 Variaciones de género, número, familia de palabras que conservan la raíz, etc.

$5 \quad$ El análisis es preliminar, nos permite identificar significados y formular interrogantes pero el mismo requiere cruce de análisis, marco teórico que sustente el mismo, etc. Las citas son transcriptas en inglés, idioma original del curso, para no perder la riqueza de las expresiones.

6 Hemos incluido datos mínimos de los protagonistas para ubicar al lector y afectar lo menos posible las normas de confidencialidad.

7 En la ejemplificación, se eligieron los textos relevantes y se transcribieron en forma parcial, a fin de no agobiar al lector con lectura innecesaria.

8 En este punto es dable aclarar que la crítica se realiza al modo en que se concibe a la ceremonia de Bar Mitzvá en el marco del judaísmo conservador, una de las corrientes centrales de la comunidad en EEUU.

$9 \quad$ Pasa del singular al plural en la respuesta.

10 Nota del autor.

11 B. es una estudiante nacida en Israel que vive actualmente en EEUU.

El método micro-textual como herramienta de análisis de foros en línea en contextos multiculturales. Marcelo I. Dorfsman 
12 Original en hebreo, la traducción es del autor.

El método micro-textual como herramienta de análisis de foros en línea en contextos multiculturales. Marcelo 